\title{
Optimization of Lipase Production by Burkholderia sp. Using Response Surface Methodology
}

\author{
Chia-Feng Lo, Chi-Yang Yu, I-Ching Kuan and Shiow-Ling Lee * \\ Department of Bioengineering, Tatung University, No 40, Sec. 3, Zhongshan N. Rd., Taipei 104, \\ Taiwan; E-Mails: alexnws@hotmail.com (C.-F.L.); chrisyu@ttu.edu.tw (C.-Y.Y.); \\ iching@ttu.edu.tw (I.-C.K.) \\ * Author to whom correspondence should be addressed; E-Mail: slee@ttu.edu.tw; \\ Tel.: +886-2-2182-2928 (ext. 6320); Fax: +886-2-2585-4735.
}

Received: 31 August 2012; in revised form: 23 October 2012 / Accepted: 8 November 2012 / Published: 13 November 2012

\begin{abstract}
Response surface methodology (RSM) was employed to optimize the extracellular lipase production by Burkholderia sp. HL-10. Preliminary tests showed that olive oil, tryptone and Tween-80 exhibited significant effects on the lipase production. The optimum concentrations of these three components were determined using a faced-centered central composite design (FCCCD). The analysis of variance revealed that the established model was significant $(p<0.01)$. The optimized medium containing $0.65 \%$ olive oil $(v / v)$, $2.42 \%$ tryptone $(w / v)$ and $0.15 \%$ Tween-80 $(v / v)$ resulted in a maximum activity of 122.3 U/mL, about three fold higher than that in basal medium. Approximately $99 \%$ of validity of the predicted value was achieved.
\end{abstract}

Keywords: lipase; Burkholderia sp.; optimization; response surface methodology

\section{Introduction}

Lipases (triacyl glycerol acylhydrolases, EC 3.1.1.3), catalyzing the hydrolysis of acylglycerides to fatty acids, di-acylglycerides, mono-acylglycerides and glycerols [1], have received considerable attention recently. They have been extensively used in food, cosmetic, textile, medical and industrial applications owing to their versatile catalytic activities of triglycerides hydrolysis, esters synthesis, trans-esterification and resolution of racemic mixtures [1-3]. Lipases were found to be produced by 
many varieties of organisms. However, lipases from microbial sources are of great importance due to their diverse commercial applications both in aqueous and non-aqueous systems [4].

Medium composition plays a crucial role in the lipase production [5]. A general practice to maximize the lipase activity is by varying one factor at a time while keeping others at constant levels. Nevertheless, this method does not depict the interactive effects among the factors and it does not locate the optimum conditions of processes. Experimental statistical techniques such as response surface methodology (RSM), however, provide an effective alternative to the conventional approach in many biotechnological processes [6-9]. RSM can reveal the correlations between the factors and responses as well as the optimum level of each factor employed [10]. Optimization of lipase production by RSM has been reported in cultures of Cadida sp. 99-125, Pseudomonas aeruginosa and Bacillus pumillus [11-13].

Burkholderia sp. HL-10 used for this study was isolated from lipid-contaminated soils [14]. Burkholderia sp., well-known for production of alkaline lipases, has been widely applied in the biodegradation of environmental pollutants [15]. Lo in his preliminary study found that medium components exhibited marked effects on lipase production by Burkholderia sp. HL-10 [14]. Herein, a central composite design of response surface approach was used to examine the interaction among medium components including olive oil, tryptone and Tween-80, and to determine their optimum concentrations in order to yield a maximum lipase activity.

\section{Results and Discussion}

\subsection{Central Composite Design and Response Surface Analysis}

Preliminary screening of medium composition indicated that olive oil, tryptone and Tween-80 exhibited significant effects on lipase production by Burkholderia sp. HL-10. One-factor-at-a-time approach was used to identify the concentration levels of these three parameters [14]. Most lipases are inducible enzymes and addition of oils proved to enhance lipase production $[13,16]$. Olive oil was the most effective compared to soybean oil, palm oil, canola oil and sunflower oil [14]. Besides, as a nitrogen source, tryptone is rich in minerals and ions which has also been reported to improve the lipase activity [17]. Tween-80 not only acted as an effective surfactant but also a lipase inducer [13]. Because RSM can serve as a successive and exploratory tool for establishing the interaction of variables [16,18], a set of 20 experiments designed with the faced-centered central composite design (FCCCD) were carried out to determine their optimum concentrations for maximum lipase production. The design matrix of the variables in coded units and actual concentrations along with the experimental response (lipase production) is presented in Table 1.

The results obtained were then subject to the analysis of variance (ANOVA) to establish a response surface quadratic model. As formulated in Equation 1, lipase activity $(Y)$ was expressed as a function of concentrations of olive oil $\left(X_{1}\right)$, tryptone $\left(X_{2}\right)$ and Tween-80 $\left(X_{3}\right)$.

$$
\begin{gathered}
Y=102.71+6.83 X_{1}+33.33 X_{2}+1.95 X_{3}+9.50 X_{1} X_{2}-0.87 X_{1} X_{3} \\
-0.60 X_{2} X_{3}-12.02 X_{1}^{2}-21.01 X_{2}^{2}+1.33 X_{2}^{3}
\end{gathered}
$$


Table 1. Face-centered central composite design of the medium components in coded and actual units for the lipase production.

\begin{tabular}{|c|c|c|c|c|c|c|c|}
\hline \multirow{2}{*}{ Run } & \multicolumn{2}{|c|}{$X_{1}$ (olive oil) } & \multicolumn{2}{|c|}{$X_{2}$ (tryptone) } & \multicolumn{2}{|c|}{$X_{3}$ (Tween-80) } & \multirow{2}{*}{$\begin{array}{l}\text { Activity } \\
\text { (U/mL) }\end{array}$} \\
\hline & coded & actual (\%) & coded & actual $(\%)$ & coded & actual (\%) & \\
\hline 1 & -1 & 0.25 & -1 & 0.5 & -1 & 0.05 & 39.3 \\
\hline 2 & 1 & 0.75 & -1 & 0.5 & -1 & 0.05 & 31.3 \\
\hline 3 & -1 & 0.25 & 1 & 2.5 & -1 & 0.05 & 81.7 \\
\hline 4 & 1 & 0.75 & 1 & 2.5 & -1 & 0.05 & 120.8 \\
\hline 5 & -1 & 0.25 & -1 & 0.5 & 1 & 0.15 & 42.6 \\
\hline 6 & 1 & 0.75 & -1 & 0.5 & 1 & 0.15 & 40.2 \\
\hline 7 & -1 & 0.25 & 1 & 2.5 & 1 & 0.15 & 91.6 \\
\hline 8 & 1 & 0.75 & 1 & 2.5 & 1 & 0.15 & 118.3 \\
\hline 9 & -1 & 0.25 & 0 & 1.5 & 0 & 0.10 & 85.5 \\
\hline 10 & 1 & 0.75 & 0 & 1.5 & 0 & 0.10 & 98.3 \\
\hline 11 & 0 & 0.50 & -1 & 0.5 & 0 & 0.10 & 45.8 \\
\hline 12 & 0 & 0.50 & 1 & 2.5 & 0 & 0.10 & 120.0 \\
\hline 13 & 0 & 0.50 & 0 & 1.5 & -1 & 0.05 & 105.2 \\
\hline 14 & 0 & 0.50 & 0 & 1.5 & 1 & 0.15 & 105.2 \\
\hline 15 & 0 & 0.50 & 0 & 1.5 & 0 & 0.10 & 97.2 \\
\hline 16 & 0 & 0.50 & 0 & 1.5 & 0 & 0.10 & 107.6 \\
\hline 17 & 0 & 0.50 & 0 & 1.5 & 0 & 0.10 & 98.3 \\
\hline 18 & 0 & 0.50 & 0 & 1.5 & 0 & 0.10 & 98.2 \\
\hline 19 & 0 & 0.50 & 0 & 1.5 & 0 & 0.10 & 101.8 \\
\hline 20 & 0 & 0.50 & 0 & 1.5 & 0 & 0.10 & 108.5 \\
\hline
\end{tabular}

As shown in Table 2, the model generated was statistically significant with a value of "Probability $>F$ " less than 0.05, suggesting the lipase activity could be well described with this model. The lack of fit measures the unfitness of the model to represent data within experimental region. Therefore, the non-significant lack-of-fit ( $p=0.5978)$ indicated the model was significant [18] and the equation was suitable for simulation of lipase production with any combination of three variables. The value of $R^{2}$ was 0.9866 revealing a relatively high correlation between experimental and predicted values and $98.66 \%$ of the variability in the response could be explained by the model [19]. The predicted $R^{2}$ of 0.9091 was in accordance with the adjusted $R^{2}$ of 0.9746 , also supporting that the regression model could be used to describe the response trends [20]. The adequate precision which measured the ratio of signal-to-noise was 26.244, higher than 4, again confirming the model adequacy [19-21]. Thus, this model could be used to navigate the design space satisfactorily.

Table 2. Analysis of variance (ANOVA) for the quadratic model of lipase activity of Burkholderia sp.

\begin{tabular}{llllll}
\hline Source & Sum of Squares & DF & Mean square & $\boldsymbol{F}$ Value & Probability $>\boldsymbol{F}$ \\
\hline Model & $1,6535.33$ & 9 & 1837.26 & 82.01 & $<0.0001$ \\
Residual & 224.03 & 10 & 22.40 & 20.82 & \\
Lack of fit & 99.02 & 5 & 19.80 & 0.79 & 0.5978 \\
Pure error & 125.01 & 5 & 25.0 & & \\
Corrected total & $1,6759.36$ & 19 & & & \\
\hline \multicolumn{5}{c}{$R^{2}=0.9866, R_{\text {pred }}^{2}=0.9091, R_{\text {adj }}^{2}=0.9746$, adequate precision $=26.244$} \\
\hline
\end{tabular}


The three-dimensional response surface curves and contour plots in Figures 1-3 showed the interactions amongst olive oil, tryptone and Tween-80. Two variables were investigated at a time while keeping the other one at a fixed concentration. Figure 1 showed a somewhat elliptical contour, suggesting a synergistic effect between olive oil and tryptone. In the range studied, higher concentrations of olive oil and tryptone markedly enhanced the production of lipase. This was consistent with the fact that lipases were generally induced in presence of oils [21,22], and tryptone was reported to enhance the lipase activity of Acinetobacter calcoaceticus [17] and Yarrowia lipolytica [23].

Figure 1. (a) Response surface curve and (b) Contour plot showing the effects of olive oil and tryptone on the lipase activity of Burkholderia sp. HL-10.

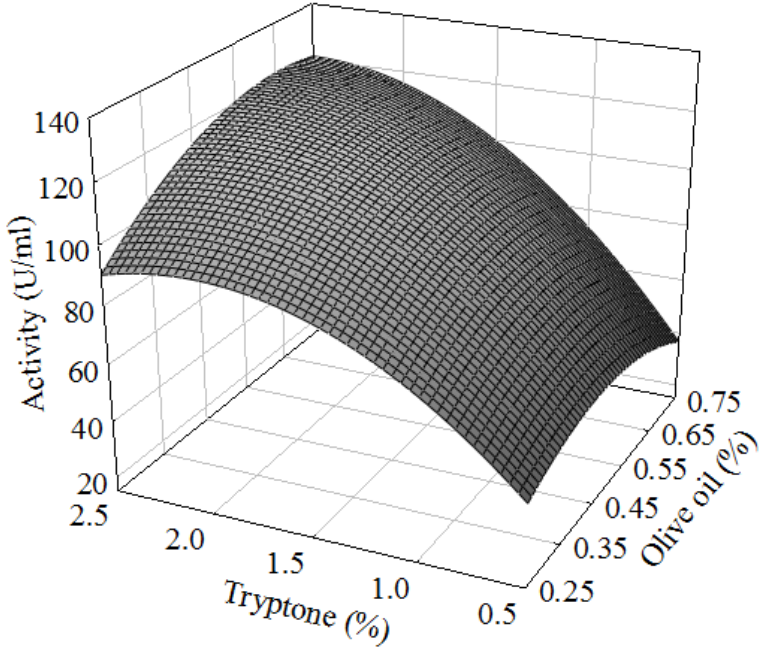

(a)

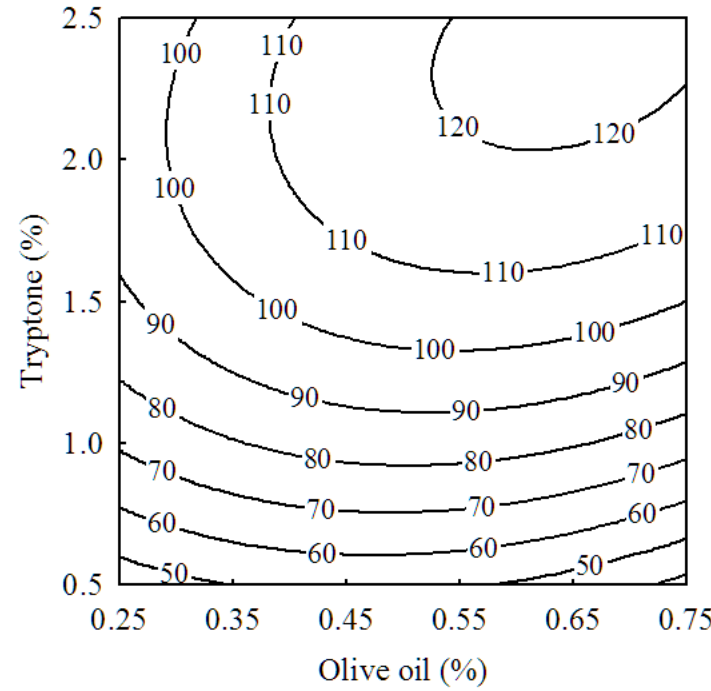

(b)

Figure 2. (a) Response surface curve and (b) contour plot showing the effects of olive oil and Tween- 80 on the lipase activity of Burkholderia sp. HL-10.

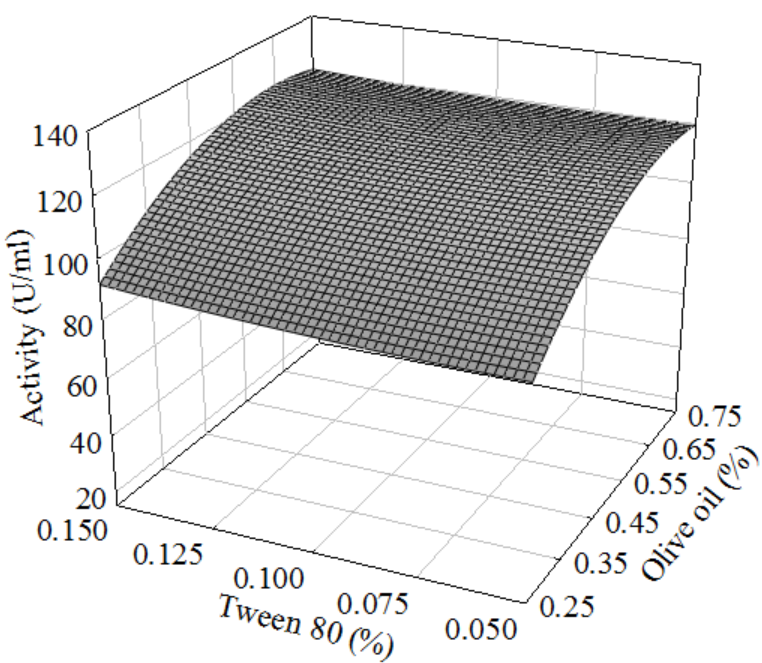

(a)

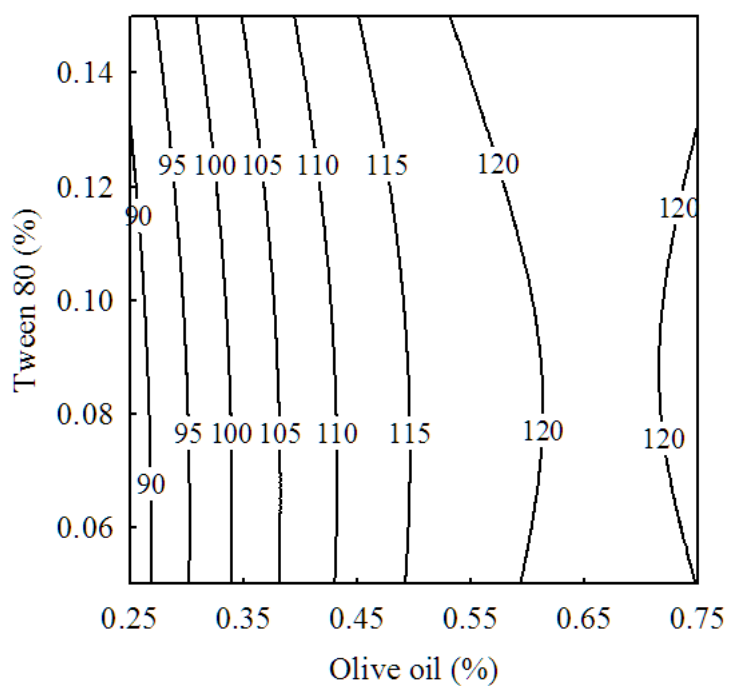

(b) 
Figure 3. (a) Response surface curve and (b) contour plot showing the effect of tryptone and Tween-80 on the lipase activity of Burkholderia sp. HL-10.

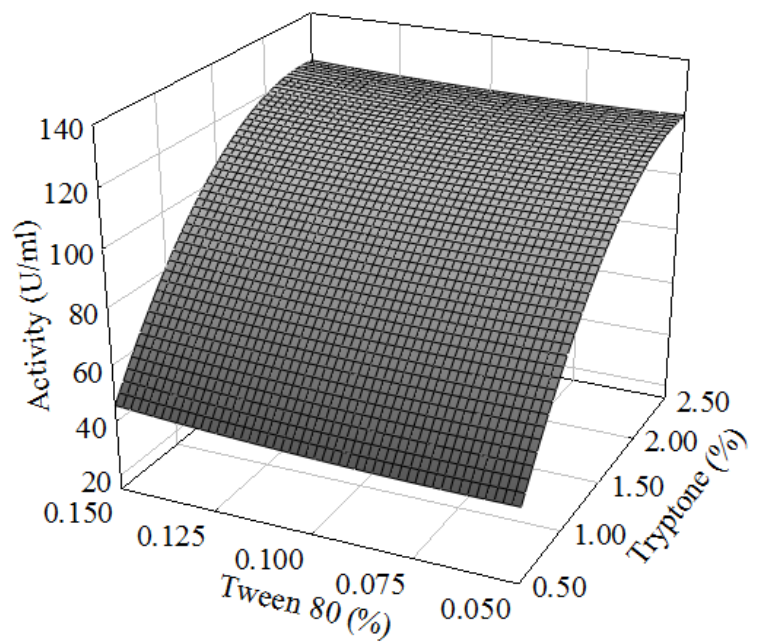

(a)

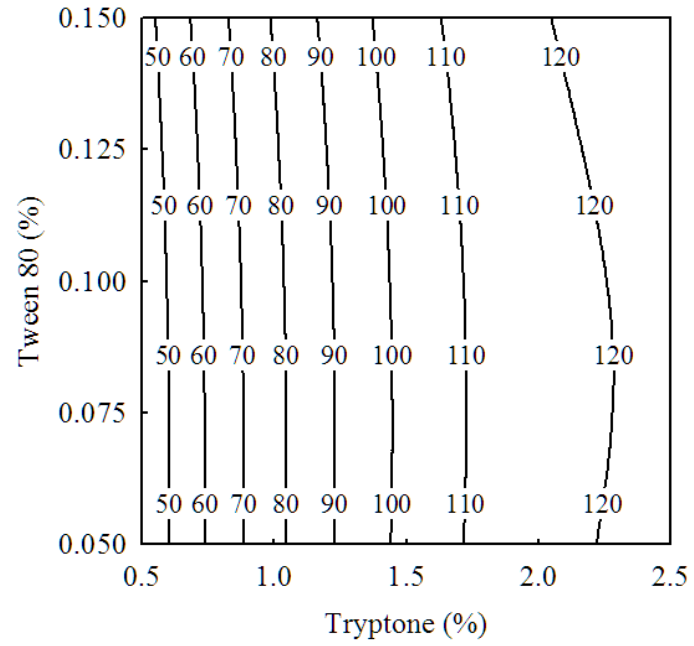

(b)

Tween-80 as a surfactant can lower the interfacial tension between oils and water and increase cell permeability, thus possibly facilitating the enzyme secretion [20]. Figure 2 showed the correlation between olive oil and Tween-80. Unexpectedly, an increase in Tween-80 concentration did not exert an obvious effect on lipase activity. It might be attributed to the lower concentration of Tween-80 of $0.05 \%$ to $0.15 \%$. In the previous reports on the lipase production by Bacillus pumilus [13], Bacillus sp. [24] and Rhizopus oligosporous [25], Tween-80 concentration ranging from $0.5 \%$ to $2 \%$ was generally added in the medium. Similar results were observed with the interactive effect between tryptone and Tween-80 (Figure 3).

The optimum concentrations for olive oil, tryptone and Tween-80 as obtained from the maximum point of the polynomial model were $0.65 \%(v / v), 2.42 \%(w / v)$ and $0.15 \%(v / v)$, respectively. In turn, the maximum activity predicted was $122.8 \mathrm{U} / \mathrm{mL}$.

\subsection{Verification of Model}

The response surface model was validated with additional experiments under the predicted conditions. The experimental value obtained was $122.3 \mathrm{U} / \mathrm{mL}$ (the average of triplicates), which was very close to the predicted value of $122.8 \mathrm{U} / \mathrm{mL}$. Approximately $99 \%$ of validity was achieved, indicating the model exerted an adequate prediction on the enzyme activity.

\subsection{Lipase Production in Basal and Optimized Media}

Figure 4 showed the time course profile of the lipase activity for Burkholderia sp. HL-10 cultivated in basal and optimized media. The lipase production started at late log phase of the bacterial growth in both media. The maximum lipase activity of $45.8 \mathrm{U} / \mathrm{mL}$ in basal medium was observed after $36-\mathrm{h}$ cultivation, while the activity maximum of $122.3 \mathrm{U} / \mathrm{mL}$ in optimized medium was achieved at $48 \mathrm{~h}$. Optimization of culture media, therefore, led to an increase in lipase yield by about three fold. The results obtained in this study were in accordance with those from previous related studies. 
Dandavate et al. [16] showed that the lipase activity of $B$. multivorans V2 was enhanced by almost 2.2 fold after optimization of the culture medium using RSM. Liu et al. [26] reported similar optimization with RSM for Burkholderia sp., which resulted in a 5-fold increase in lipase production. RSM was also performed for Bacillus pumilus [13] and Rhizopus delemar [20], giving rise to an increase in lipase production by 3.14 and 3.25 fold, respectively.

Figure 4. Lipase production by Burkholderia sp. in basal and optimized media.

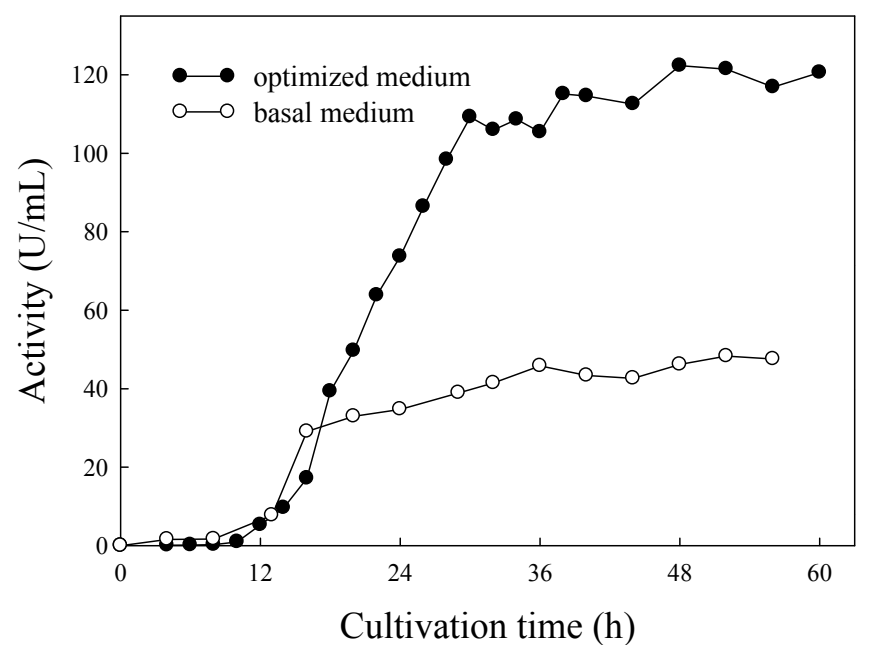

\section{Experimental Section}

\subsection{Bacterial Strain and Preparation of Inoculum}

The bacteria strain used in this study was isolated from lipid-contaminated soil samples collected in Tatung University. Identification was carried out by morphological and biochemical methods and identified as Burkholderia sp. [14]. The organism was grown on Luria-Bertani (LB) agar slant which consisted of $1 \%$ tryptone $(w / w), 1 \% \mathrm{NaCl}(w / w), 0.5 \%$ yeast extract $(w / w)$ and $1.5 \%$ agar $(w / w)$. The slants were incubated at $30{ }^{\circ} \mathrm{C}$ for $24 \mathrm{~h}$. The inoculum was prepared by aseptically transferring a loop full of cells from the agar slant into $50 \mathrm{~mL}$ LB medium. The cultivation was then carried out at $30{ }^{\circ} \mathrm{C}$ on an orbital shaker at $200 \mathrm{rpm}$ for $10 \mathrm{~h}$ to yield a microbial density of $c a .10^{9} \mathrm{CFU} / \mathrm{mL}$.

\subsection{Culture Conditions for Lipase Production}

Two hundred microliters of the inoculum prepared above was added to $50 \mathrm{~mL}$ medium in a $250 \mathrm{~mL}$ Erlenmeyer flask. The basal medium for lipase production consisted of $1 \%(v / v)$ soybean oil, $1 \%(w / v)$ tryptone, $0.1 \%(v / v)$ Tween $80,0.02 \%(w / v)$ yeast extract, $0.02 \%(w / v) \mathrm{NaNO}_{3}$ and $0.02 \%(w / v)$ $\mathrm{MgSO}_{4}$ with a pH of 6.5 adjusted by $1 \mathrm{M} \mathrm{HCl}$ [15]. According to the results obtained from one-factor-at-a-time approach, the medium employed in the design matrix of FCCCD was composed of $0.5 \%$ olive oil, $1.5 \%(w / v)$ tryptone, $0.1 \%(v / v)$ Tween-80, $0.06 \%(w / v)$ yeast extract, $0.02 \%(w / v)$ $\mathrm{NH}_{4} \mathrm{CH}_{3} \mathrm{COO}$ and $0.05 \%(w / v) \mathrm{MgSO}_{4}$ [14]. The culture was incubated at $30{ }^{\circ} \mathrm{C}$ with orbital shaking at $200 \mathrm{rpm}$ for $48 \mathrm{~h}$. The lipase activity in the culture supernatant was then measured after centrifugation at $12,500 \mathrm{~g}$ for $10 \mathrm{~min}$ at $4{ }^{\circ} \mathrm{C}$. 


\subsection{Response Surface Methodology}

The statistical approach using FCCCD developed by the Design Expert software (Version 6.0.1, Stat-Ease Inc., Minneapolis, USA) was used to generate a set of 20 experimental runs with six replicated center points. Three different levels, low $(-1)$, medium $(0)$ and high $(+1)$ were used to study the independent variables (olive oil, tryptone and Tween-80), and the lipase activity was taken as a dependent variable $(Y)$ (Table 1). A quadratic polynomial regression model was established to describe the relationship between dependent and independent variables:

$$
Y=\beta_{0}+\beta_{1} X_{1}+\beta_{2} X_{2}+\beta_{3} X_{3}+\beta_{12} X_{1} X_{2}+\beta_{13} X_{1} X_{3}+\beta_{23} X_{2} X_{3}+\beta_{11} X_{1}^{2}+\beta_{22} X_{2}^{2}+\beta_{33} X_{3}^{2}
$$

where $Y$ is the predicted response (lipase activity); $X_{1}, X_{2}$ and $X_{3}$ are the independent variables (olive oil, tryptone and Tween- 80 concentrations); $\beta_{0}$ is the intercept, $\beta_{0}, \beta_{1}$ and $\beta_{3}$ are the linear coefficient; $\beta_{12}, \beta_{13}$ and $\beta_{23}$ are the interaction coefficient; $\beta_{11}, \beta_{22}$ and $\beta_{33}$ are the quadratic coefficients. Analysis of variance (ANOVA) was performed to evaluate the adequacy of the model. Three-dimensional response surface curves were plotted by Design Expert software to display the interaction among various variables.

\subsection{Assay for Lipase Activity}

Lipase activity was monitored using $p$-nitrophenyl palmitate ( $p$-NPP) as substrate according to the method described by Pencreac'h and Baratti [27] with minor modification. The assay mixture was prepared by adding $90 \mu \mathrm{L}$ of $8.25 \mathrm{mM} p$-NPP in isopropanol and $810 \mu \mathrm{L}$ of solution containing $50 \mathrm{mM}$ Tris- $\mathrm{HCl}$ buffer $(\mathrm{pH} 8.0$ ) with $0.494 \%$ Triton $\mathrm{X}-100$ and $0.12 \%$ arabic gum, followed by pre-incubation at $40{ }^{\circ} \mathrm{C}$ for $5 \mathrm{~min}$. The enzymatic reaction was then initiated by adding $100 \mu \mathrm{L}$ of appropriate diluted lipase sample (culture supernatant) and proceeded at $40{ }^{\circ} \mathrm{C}$ for $5 \mathrm{~min}$. The amount of $p$-nitrophenol released was measured at $410 \mathrm{~nm}$ using a Helios- $\alpha$ spectrophotometer equipped with a thermostatic cell holder (Unicam, Cambridge, UK). One unit (U) of lipase activity is defined as the amount of enzyme that liberates $1 \mu \mathrm{mol} p$-nitrophenol per minute at $40{ }^{\circ} \mathrm{C}$. All experiments were performed in triplicate.

\section{Conclusions}

The present work aimed to optimize lipase production by Burkholderia sp. HL-10 with response surface methodology. The $R^{2}$ value of 0.9866 for the model established clearly indicated a relatively high correlation between model and experimental data. The optimum concentrations of olive oil, tryptone and Tween- 80 were determined to be $0.65 \%(v / v), 2.42 \%(w / v)$ and $0.15 \%(v / v)$, respectively, which gave rise to almost a three-fold increase in the maximum lipase activity. Thus, this work demonstrated the feasibility of statistical methodology to develop optimum medium composition with a minimum number of experimental trials.

\section{Acknowledgments}

The financial support by the grant (B99-S01-027) from Tatung University was acknowledged. 


\section{References}

1. Houde, A.; Kademi, A.; Leblanc, D. Lipases and their industrial applications. Appl. Biochem. Biotechnol. 2004, 118, 155-170.

2. Ribeiro, B.D.; de Castro, A.M.; Coelho, M.A.Z.; Freire, D.M.G. Production and use of lipases in bioenergy: A review from the feedstocks to biodiesel production. Enzyme Res. 2011, 2011, 1-16.

3. Hasan, F.; Shah, A.A.; Javed, S.; Hameed, A. Enzymes used in detergents: Lipases. Afr. J. Biotechnol. 2010, 9, 4836-4844.

4. Hasan, F.; Shah, A.A.; Hameed, A. Industrial applications of microbial lipases. Enzyme Microb. Technol. 2006, 39, 235-251.

5. Ghosh, P.K.; Saxena, R.K.; Gupta, R.; Yadav, R.P.; Davidson, S. Microbial lipases: Production and applications. Sci. Prog. 1996, 79, 119-157.

6. Soto-Cruz, O.; Saucedo-Castañeda, G.; Pablos-Hach, J.L.; Gutiérrez-Rojas, M.; Favela-Torres, E. Effect of substrate composition on the mycelial growth of Pleurotus ostreatus. An analysis by mixture and response surface methodologies. Process Biochem. 1999, 35, 127-133.

7. Li, Y.; Cui, F.; Liu, Z.; Xu, Y.; Zhao, H. Improvement of xylanase production by Penicillium oxalicum ZH-30 using response surface methodology. Enzym. Microb. Technol. 2007, 40, 1381-1388.

8. Kumari, A.; Mahapatra, P.; Banerjee, R. Statistical optimization of culture conditions by response surface methodology for synthesis of lipase with Enterobacter aerogenes. Braz. Arch. Biol. Technol. 2009, 52, 1349-1356.

9. Yin, X.; You, Q.; Jiang, Z. Optimization of enzyme assisted extraction of polysaccharides from Tricholoma matsutake by response surface methodology. Carbohydr. Polym. 2011, 86, 1358-1364.

10. Gupta, N.; Mehra, G.; Gupta, R. A glycerol-inducible thermostable lipase from Bacillus sp.: Medium optimization by a Plackett-Burman design and by response surface methodology. Can. J. Microbiol. 2004, 50, 361-368.

11. He, Y.-Q.; Tan, T.-W. Use of response surface methodology to optimize culture medium for production of lipase with Candida sp. 99-125. J. Mol. Catal. B Enzym. 2006, 43, 9-14.

12. Ruchi, G.; Anshu, G.; Khare, S.K. Lipase from solvent tolerant Pseudomonas aeruginosa strain: Production optimization by response surface methodology and application. Bioresour. Technol. 2008, 99, 4796-4802.

13. Kumar, R.; Mahajan, S.; Kumar, A.; Singh, D. Identification of variables and value optimization for optimum lipase production by Bacillus pumilus RK31 using statistical methodology. New Biotechnol. 2011, 28, 65-71.

14. Lo, C.-F. Optimal production of lipase from Burkholderia sp. Master's Thesis, Tatung University. Taipei, Taiwan, 2012.

15. Rathi, P.; Saxena, R.K.; Gupta, R. A novel alkaline lipase from Burkholderia cepacia for detergent formulation. Process Biochem. 2001, 37, 187-192.

16. Dandavate, V.; Jinjala, J.; Keharia, H.; Madamwar, D. Production, partial purification and characterization of organic solvent tolerant lipase from Burkholderia multivorans V2 and its application for ester synthesis. Bioresour. Technol. 2009, 100, 3374-3381. 
17. Cordenons, A.; Gonzalez, R.; Kok, R.; Hellingwerf, K.J.; Nudel, C. Effect of nitrogen sources on the regulation of extracellular lipase production in Acinetobacter calcoaceticus strains. Biotechnol. Lett. 1996, 18, 633-638.

18. Sifour, M.; Zaghloul, T.I.; Saeed, H.M.; Berekaa, M.M.; Abdel-Fattah, Y.R. Enhanced production of lipase by the thermophilic Geobacillus stearothermophilus strain-5 using statistical experimental designs. New Biotechnol. 2010, 27, 330-336.

19. Shabbiri, K.; Adnan, A. Bio-statistically optimized production of lipases by Brevibacterium linens DSM 20158. World Appl. Sci. J. 2011, 13, 1059-1066.

20. Açıkel, Ü.; Erşan, M.; Sağ Açıkel, Y. Optimization of critical medium components using response surface methodology for lipase production by Rhizopus delemar. Food Bioprod. Process. 2010, 88, 31-39.

21. Gupta, N.; Sahai, V.; Gupta, R. Alkaline lipase from a novel strain Burkholderia multivorans: Statistical medium optimization and production in a bioreactor. Process Biochem. 2007, 42, 518-526.

22. Lima, V.M.G.; Krieger, N.; Sarquis, M.I.M. Effect of nitrogen and carbon sources on lipase production by Penicillium aurantiogriseum. Food Technol. Biotechnol. 2003, 41, 105-110.

23. Turki, S.; Kraeim, I.B.; Weeckers, F.; Thonart, P.; Kallel, H. Isolation of bioactive peptides from tryptone that modulate lipase production in Yarrowia lipolytica. Bioresour. Technol. 2009, 100, 2724-2731.

24. Sidhu, P.; Sharma, R.; Soni, S.; Gupta, J. Production of extracellular alkaline lipase by a new thermophilic Bacillus sp. Folia Microbiol. 1998, 43, 51-54.

25. Iftikhar, T.; Athar, H. Effects of nutrients on the extracellular lipase production by mutant strain of Rhizopus oligosporous TUV-31. Biotechnology 2002, 1, 15-20.

26. Liu, C.-H.; Lu, W.-B.; Chang, J.-S. Optimizing lipase production of Burkholderia sp. by response surface methodology. Process Biochem. 2006, 41, 1940-1944.

27. Pencreac'h, G.; Baratti, J.C. Activity of Pseudomonas cepacia lipase in organic media is greatly enhanced after immobilization on a polypropylene support. Appl. Microbiol. Biotechnol. 1997, 47, 630-635.

(C) 2012 by the authors; licensee MDPI, Basel, Switzerland. This article is an open access article distributed under the terms and conditions of the Creative Commons Attribution license (http://creativecommons.org/licenses/by/3.0/). 